\title{
Investigation of thermally evaporated nanocrystalline thin cobalt films
}

\author{
W. Kozłowski ${ }^{1}$ J. Balcerski ${ }^{1}$ P. J. Kowalczyk ${ }^{1} \cdot$ M. Cichomski $^{2} \cdot$ W. Szmaja ${ }^{1}$
}

Received: 18 October 2016 / Accepted: 16 January 2017 / Published online: 15 February 2017

(c) The Author(s) 2017. This article is published with open access at Springerlink.com

\begin{abstract}
In this paper, a study has been made of nanocrystalline thin cobalt films with thicknesses in the range from 10 to $60 \mathrm{~nm}$. The films were thermally evaporated at incidence angle of $0^{\circ}$ in a vacuum of about $10^{-5}$ mbar. The morphological structure of the films consists of nanocrystalline grains regular in shape and densely packed. As the film thickness is increased from 10 to $60 \mathrm{~nm}$, the average grain size increases from 22.0 to $28.9 \mathrm{~nm}$. The films crystallize mainly in the hexagonal close-packed phase of cobalt. The magnetic structure is composed of domains. In films with thicknesses in the range from 10 to $40 \mathrm{~nm}$, the domains are magnetized in the plane of the film, while films with thicknesses of 50 and $60 \mathrm{~nm}$ possess both inplane and perpendicular magnetization components. The domains with inplane magnetization are irregular in shape and typically from a few to $10 \mathrm{~mm}$ in size, whereas the domains with perpendicular magnetization form a fine maze stripe pattern of the order of $100 \mathrm{~nm}$ in width.
\end{abstract}

\section{Introduction}

The morphological and magnetic domain structures determine the properties of magnetic specimens. Therefore, a detailed knowledge and complete understanding of the mentioned structures and the correlation between these

W. Kozłowski

wkozl@std2.phys.uni.lodz.pl

1 Department of Solid State Physics, Faculty of Physics and Applied Informatics, University of Łódź, Pomorska 149/153, 90-236 Łódź, Poland

2 Department of Materials Technology and Chemistry, Faculty of Chemistry, University of Lodz, Pomorska 163, 90-236 Lodz, Poland structures are of large importance. From the practical point of view, they are very significant for tailoring of the specimens with the required properties. In particular, they allow to develop high-density magnetic and magneto-optic recording media, modern recording heads, high-performance permanent magnets, magnetic sensors, and transformer steel sheets $[1,2]$. The knowledge of the magnetic domain behavior in relation to the morphological structure of the specimens is also important for theoretical modeling of magnetic properties [1,2].

Thin magnetic films attract large attention from the fundamental as well as technological point of view. On the fundamental side, they exhibit different magnetic properties, such as magnetic anisotropy [3], magnetic microstructure [4], coercivity [5], and magnetoresistance [6], depending on their thickness, composition, crystalline structure, and preparation conditions. From the technological point of view, thin magnetic films have a number of applications; for example, they are used in magnetic information storage media, magneto-optic recording media, magnetic devices, and sensors $[1,2]$.

In particular, cobalt thin films have been the subject of wide and intense study in recent years. They can be prepared by various techniques, for example by sputtering, thermal evaporation, electron beam evaporation, molecular beam epitaxy, pulsed laser deposition, electrodeposition, and chemical vapor deposition [7-9]. The obtained films are usually nanocrystalline in nature and possess specific, improved mechanical, physical, magnetic, and chemical properties in comparison with conventional microcrystalline counterparts. Depending on the film thickness as well as the preparation method and conditions used, cobalt thin films exhibit a wide range of morphological and magnetic properties, and especially various magnetic domain structures with inplane and outofplane magnetization [10-13]. 
The applications of cobalt thin films include, for example, spintronic devices, such as spin valves, magnetic tunnel junctions and magnetic random access memories, microelectromechanical systems, and logic devices and circuits [14-17]. Recently, thin Co films have been also used to investigate small and isolated skyrmions [18] which presence can be associated with logical one state and in consequence used to construct novel memories [19].

This paper presents an investigation of thermally evaporated nanocrystalline thin cobalt films with thicknesses in the range from 10 to $60 \mathrm{~nm}$. The morphological structure of the films was made visible using atomic force microscopy (AFM), while their magnetic domain structure was imaged with magnetic force microscopy (MFM) and the Fresnel mode of transmission electron microscopy (TEM). To the authors' knowledge, such study is reported for the first time. Our results clearly show that the magnetization for these films changes from in plane for $10 \mathrm{~nm}$-thick films to mainly perpendicular one for $60 \mathrm{~nm}$ films. Results of these studies are of high importance for design of novel highdensity magnetic storage media and magneto-optic devices.

\section{Experimental}

The investigated nanocrystalline cobalt films possessed different thicknesses in the range from 10 to $60 \mathrm{~nm}$. They were thermally evaporated at an incidence angle of $0^{\circ}$ (with respect to the surface normal) in a vacuum of approximately $10^{-5}$ mbar. For these experiments, PREVAC EF $40 \mathrm{C} 1$ Effusion Cell was used with $\mathrm{Al}_{2} \mathrm{O}_{3}$ crucible and currents up to $10 \mathrm{~A}$. The film thicknesses were determined by a quartz crystal microbalance. The films were deposited simultaneously on unheated glass substrates and $\mathrm{NaCl}$ crystals; the latter films were prepared for the purpose of studying by TEM. For AFM and MFM measurements, glass substrate was used because of its non-magnetic nature, smoothness, and uniformity which makes it ideal for device applications [20].

The distribution of magnetic domains and the nature of domain walls in the investigated films were determined by the Fresnel (or defocus) mode of TEM [21, 22] using a Tesla BS 540 instrument. For this purpose, the $\mathrm{NaCl}$ substrates were dissolved away in water and then the films were caught on microscope copper grids. Our investigations [22, 23] and those reported by other authors [24, 25] show that the film structure is practically not affected by the specimen preparation method for TEM observation. The magnetic microstructure was also imaged with MFM using an NTMDT NTEGRA Prima instrument operated in the dynamic mode. We used Bruker MESPLC, MESPLM, and MESPHM magnetic probes. The tips were magnetized along their axis by a permanent magnet prior to the measurements. The tip axis was perpendicular to the specimen surface. The image signal was detected as the phase shift of an oscillating cantilever. The morphological structure of the films was revealed with AFM using an NTMDT NTEGRA Prima instrument characterized among others by piezo creeping compensation feedback loop (which prevents introduction of artifacts in recorded images). The crystallographic structure of the films was investigated using the selected area electron diffraction (SAED) patterns of TEM.

\section{Results and discussion}

Figure 1a-f shows AFM images of the morphological structure of cobalt films $10 \mathrm{~nm}$ (a), $20 \mathrm{~nm}$ (b), $30 \mathrm{~nm}$ (c), $40 \mathrm{~nm}$ (d), $50 \mathrm{~nm}$ (e), and $60 \mathrm{~nm}$ (f) in thickness. All the images were taken from $500 \cdot 500 \mathrm{~nm}$ area. For each sample, several upward and downward scans were compared to eliminate scanning artifacts. As expected, the morphological structure consists of grains. The grains are regular in shape and possess small sizes in nanometer range. In all the studied films, the grains are packed densely. It can also be seen that the grain size increases with increasing film thickness. Note that for some images recorded at the scale presented in Fig. 1, some geometric alignment and preferred elongation of the grains can be noticed (Fig. 1a, f). Nevertheless, for images taken from sufficiently large film surface area (larger than about $2 \times 2 \mu \mathrm{m}$ ), no geometric alignment and no preferred elongation of the grains were generally observed, in agreement with expectations.

The root-mean-square (rms) values of the surface roughness for different film thicknesses were determined. For each film thickness, the average data were obtained using $500 \times 500 \mathrm{~nm}$ AFM images taken at various places on the film surface. The average surface roughness as a function of the film thickness for cobalt films is presented in Fig. 2. As the film thickness is increased from 10 to $40 \mathrm{~nm}$, the average surface roughness increases from 0.44 to $0.89 \mathrm{~nm}$, and for 50 and $60 \mathrm{~nm}$-thick films, it is practically the same as that for $40 \mathrm{~nm}$-thick films.

The average grain sizes for the studied films were obtained by the standard linear intercept method [26, 27]. The dependence of the average grain size on the film thickness for cobalt films is shown in Fig. 3. As the film thickness is increased from 10 to $60 \mathrm{~nm}$, the average grain size increases from 22.0 to $28.9 \mathrm{~nm}$. In this context, it is worth noting that the observed increase in the average grain size with increasing film thickness is generally consistent with the results reported in Refs [28-30].

Figure 4 presents the electron diffraction pattern of TEM for a cobalt film $40 \mathrm{~nm}$ in thickness. Such patterns were observed for all the investigated films. The occurrence of 

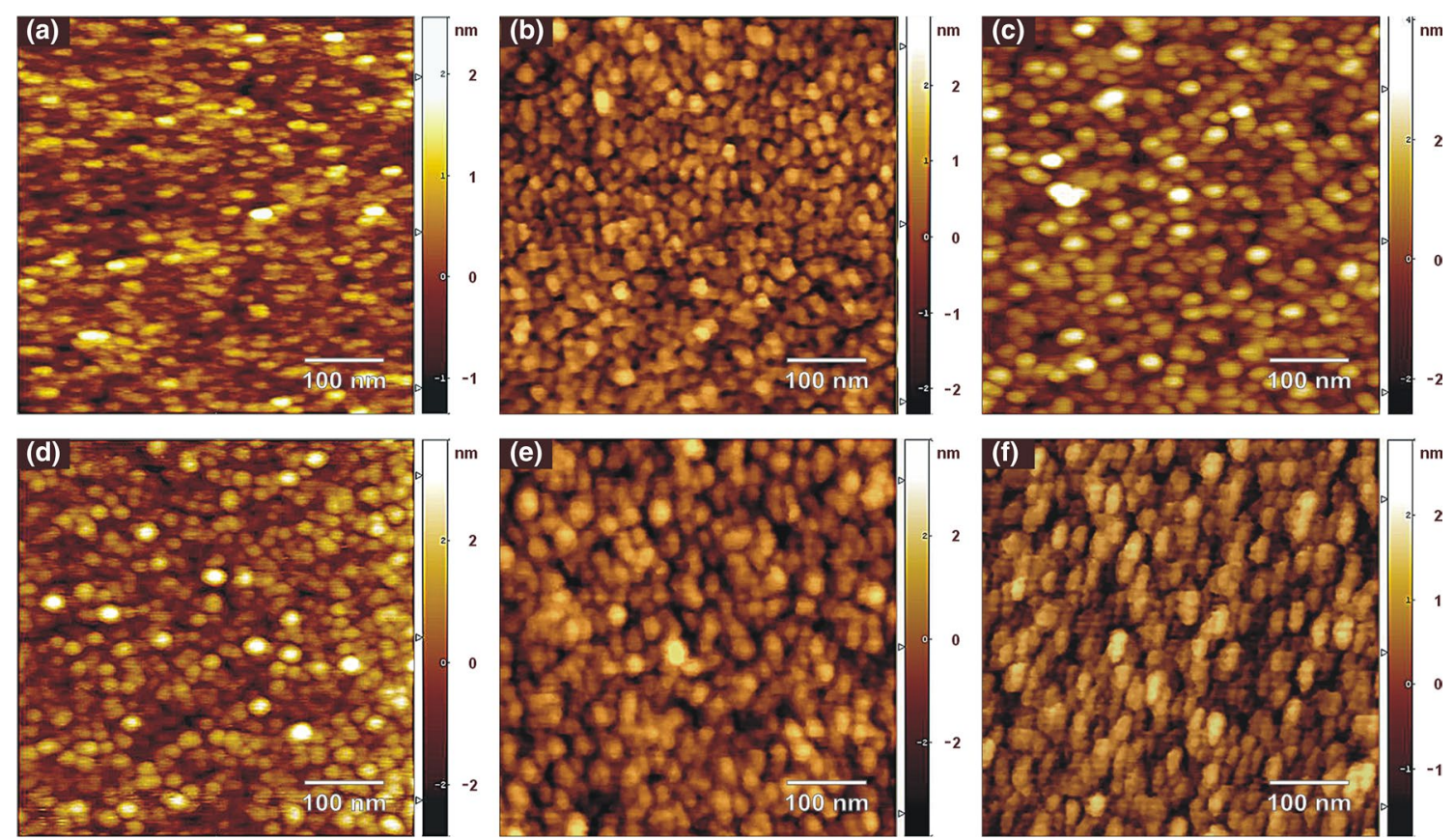

Fig. 1 AFM images of the morphological structure of $10 \mathrm{~nm}(\mathbf{a}), 20 \mathrm{~nm}(\mathbf{b}), 30 \mathrm{~nm}(\mathbf{c}), 40 \mathrm{~nm}(\mathbf{d}), 50 \mathrm{~nm}(\mathbf{e})$, and $60 \mathrm{~nm}(\mathbf{f})$-thick cobalt films

Fig. 2 Dependence of the average surface roughness on the film thickness for cobalt films

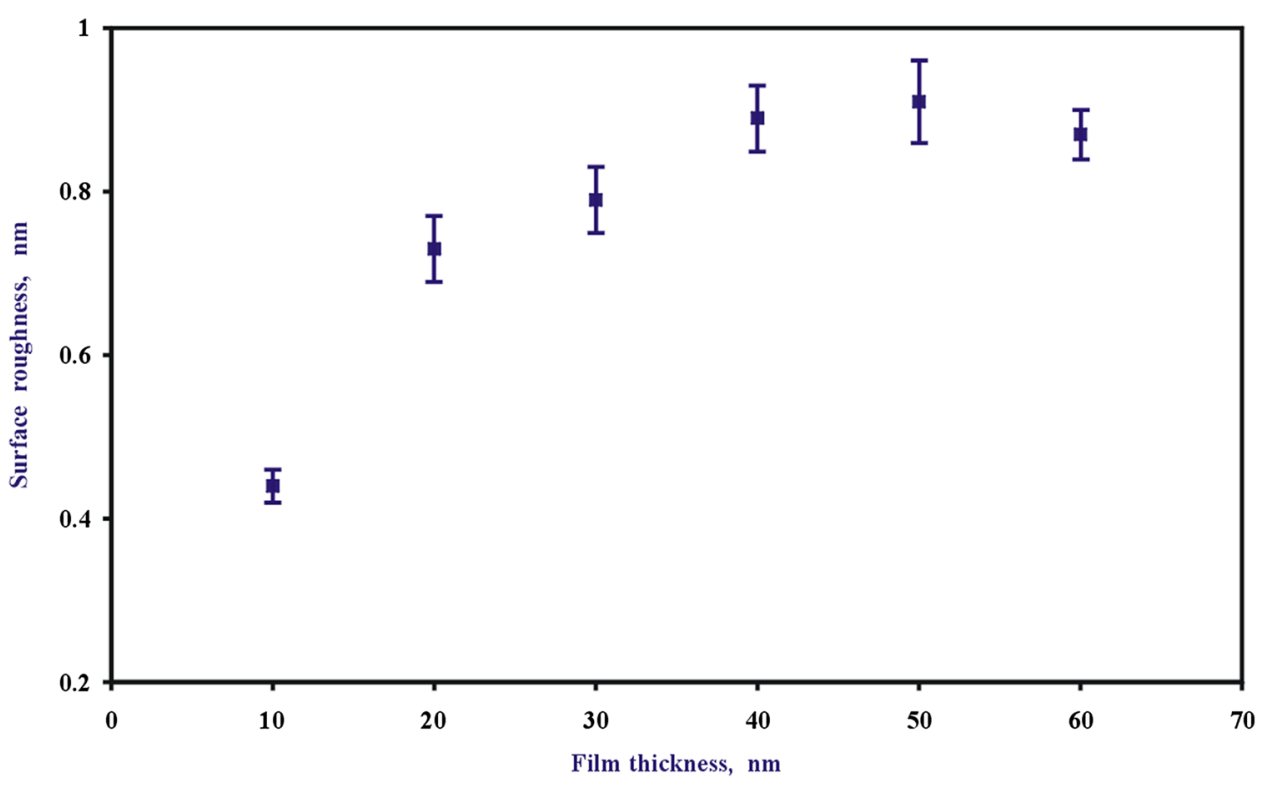

continuous diffraction rings proves that the films are polycrystalline in character. In addition, the set of three diffraction rings representing the (0110), (0002), and (011) planes, observed with clarity and high intensity shows that the films are mainly composed of the hexagonal closepacked (HCP) phase of cobalt. Moreover, the analysis of the relative intensities of the observed rings exhibits the presence of (0002) texture where the hexagonal axis is preferentially oriented perpendicularly to the film surface
[31]. Nevertheless, to get a deeper insight into the crystallographic orientation of the films, it is necessary to apply more sensitive techniques, such as X-ray diffraction (XRD) $\Theta-2 \Theta$ spectra, [12, 28, 29], the X-ray Schulz pole figures $[12,32]$, or the electron backscattered diffraction (EBSD) method $[33,34]$. These measurements are planned in near future.

Figure 5a-f shows images of the magnetic microstructure of $10 \mathrm{~nm}$ (a), $20 \mathrm{~nm}$ (b), $30 \mathrm{~nm}$ (c), $40 \mathrm{~nm}$ (d), $50 \mathrm{~nm}$ 
Fig. 3 Dependence of the average grain size on the film thickness for cobalt films

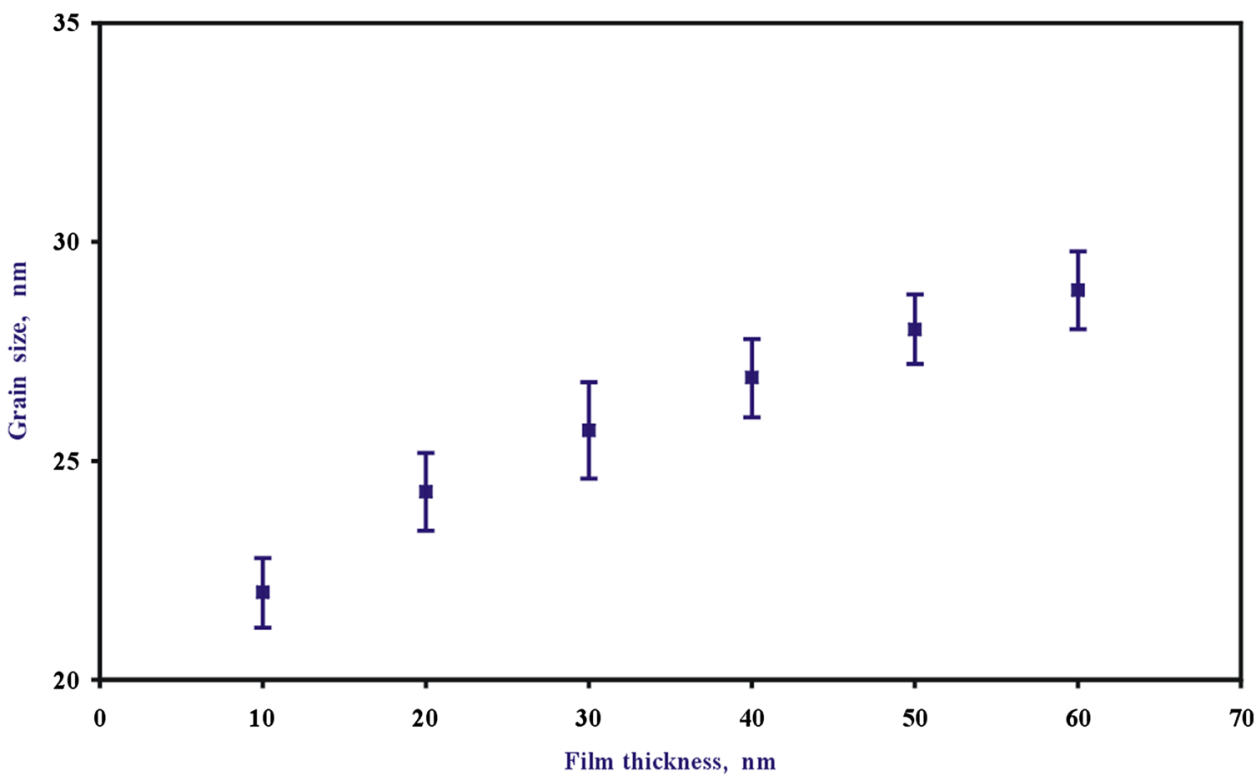

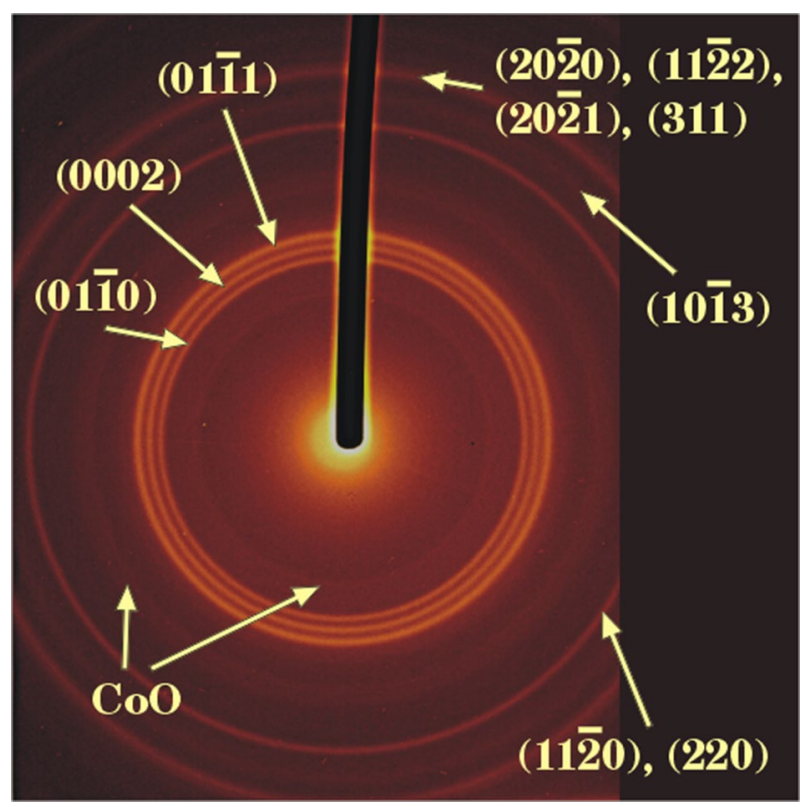

Fig. 4 Typical TEM diffraction pattern for cobalt films with thickness below $60 \mathrm{~nm}$

(e), and $60 \mathrm{~nm}$ (f)-thick cobalt films, recorded with the Fresnel mode of TEM. In $10 \mathrm{~nm}$-thick films, only Néel type walls were present (Fig. 5a). In 20 and 30 nm-thick films, the occurrence of Néel type as well as cross-tie walls was observed (Fig. 5b, c). For the film thickness in the range from 40 to $60 \mathrm{~nm}$, only cross-tie walls occurred (Fig. 5d-f). It is also to be noted that, in general, the distribution of magnetization for all the investigated films, revealed by the Fresnel mode of TEM, is found to be isotropic in the plane of the film.
The cross-tie wall (observed for Co films characterized by thickness $\geq 20 \mathrm{~nm}$ ) constitutes a transition between Néel and Bloch type walls. In the case of the cross-tie wall, the cross ties serve to decrease the demagnetizing energy and are imaged by the conventional Bitter pattern technique and the Fresnel mode of TEM as bars perpendicular to the main wall [35-38]. It has to be noted that observation of the cross-tie wall for $20 \mathrm{~nm}$-thick films is quite unusual (mostly such wall type shows up for film thicknesses in the range $30-40 \mathrm{~nm}$ and is observed up to $100 \mathrm{~nm}$ ). We are aware of only three previous papers reporting observation of crosstie wall for film thinner than $20 \mathrm{~nm}$, i.e., Donnet et al. [12] reported their formation in $20 \mathrm{~nm}$-thick cobalt films, Gentils et al. in $17.5 \mathrm{~nm}$-thick permalloy films [39], and Szmaja et al. in $10 \mathrm{~nm}$-thick permalloy films [22].

In the present paper, as previously mentioned, we report the coexistence of Néel type and cross-tie walls in 20 and $30 \mathrm{~nm}$-thick films (Fig. 5b, c). The cause for the presence of Néel type as well as cross-tie walls is that their wall energies are comparable at the mentioned film thicknesses. According to the theoretical works of Metlov [40, 41], the film thickness corresponding to the transition between the Néel and cross-tie walls is about $8 \mathrm{~nm}$. Considering the simplifications used in the theory, this theoretical result is found to be in reasonable agreement with our experimental observations.

In the case of the studied cobalt films, the cross-tie walls in images recorded with the Fresnel mode of TEM can, in fact, be recognized by the presence of the circular Bloch lines, which are narrowly spaced and clearly visible as dark filled circles (Fig. 5b-f). This is because the cross ties are very short and consequently hardly visible or practically invisible in the images. The small cross-tie spacing and 

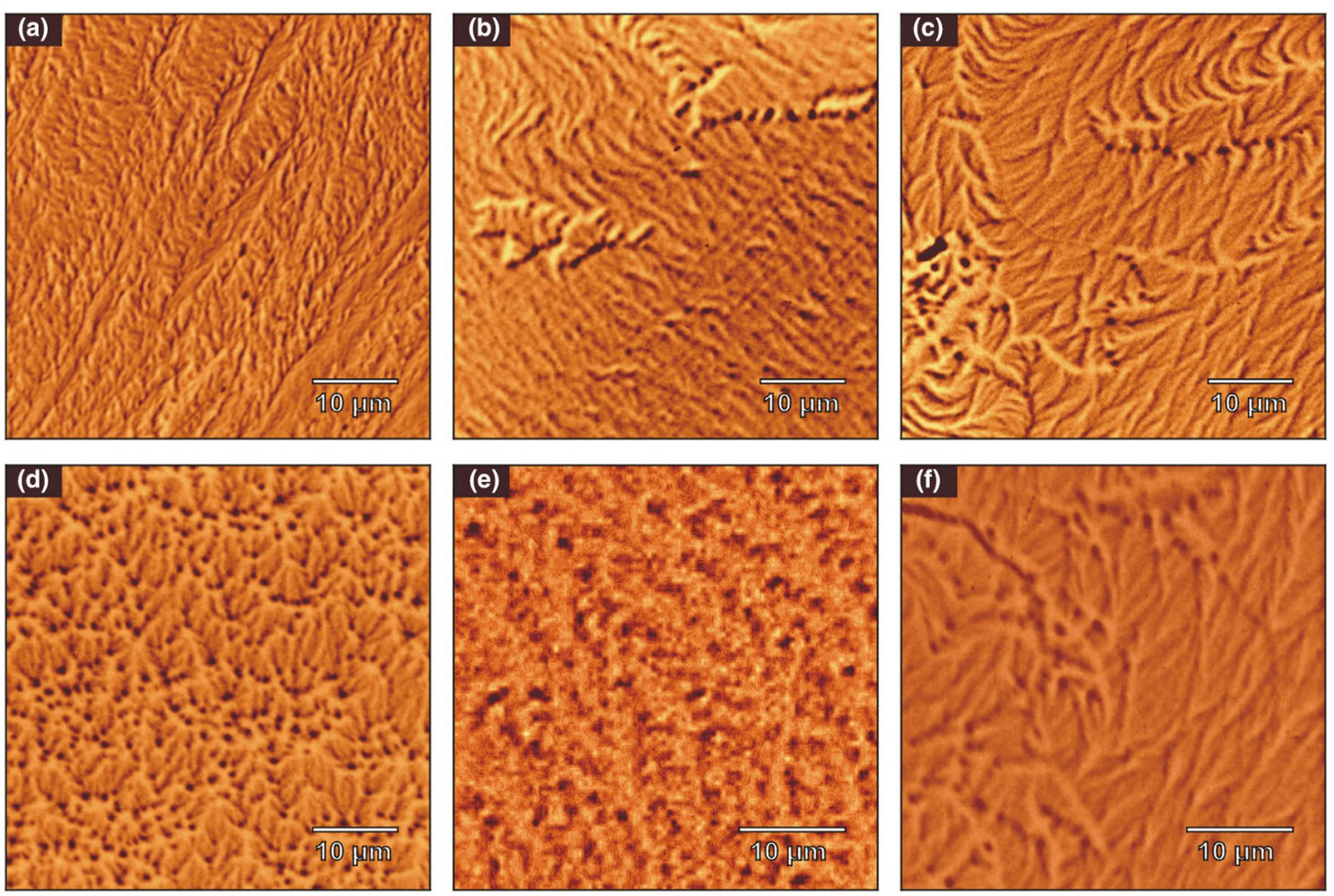

Fig. 5 Images of the magnetic microstructure of $10 \mathrm{~nm}(\mathbf{a}), 20 \mathrm{~nm}(\mathbf{b}), 30 \mathrm{~nm}(\mathbf{c}), 40 \mathrm{~nm}(\mathbf{d}), 50 \mathrm{~nm}(\mathbf{e})$, and $60 \mathrm{~nm}(\mathbf{f})$ thick cobalt films, recorded by the Fresnel mode of TEM

length are due to the high magnetic anisotropy of cobalt in the HCP phase (the magnetocrystalline anisotropy constant is $K_{\mathrm{Co}}=4.5 \times 10^{5} \mathrm{~J} / \mathrm{m}^{3}$ ). According to the theoretical considerations of Middelhoek [35], the cross-tie spacing and length are inversely proportional to the magnetic anisotropy constant. This theoretical prediction appears to be confirmed by the images of the magnetic microstructure shown in Fig. 5b-f. In Fig. 6, we present an image of the magnetic microstructure of a $40 \mathrm{~nm}$-thick permalloy film, recorded by the Fresnel mode of TEM. This permalloy film was thermally evaporated using the same preparation conditions as those for cobalt films [22]. The magnetocrystalline anisotropy constant of permalloy is $K_{\mathrm{py}}=100 \mathrm{~J} / \mathrm{m}^{3}$, i.e., it is much smaller than that of cobalt. Thus, as predicted by Middelhoek [35], one expects that the cross-tie walls in permalloy should have much larger spacing and length in comparison with cobalt. And, indeed, the experiment confirms very nicely the theoretical prediction (compare Figs. 5d, 6).

The ripple structure of the magnetization was clearly seen in all images of cobalt films recorded with the Fresnel mode of TEM (Fig. 5a-f). The ripple structure is directly related to the polycrystalline character of the studied films and is due to local variations of the magnetic anisotropy $[39,42]$. The ripple direction is always oriented perpendicular to the magnetization direction and hence the magnetic

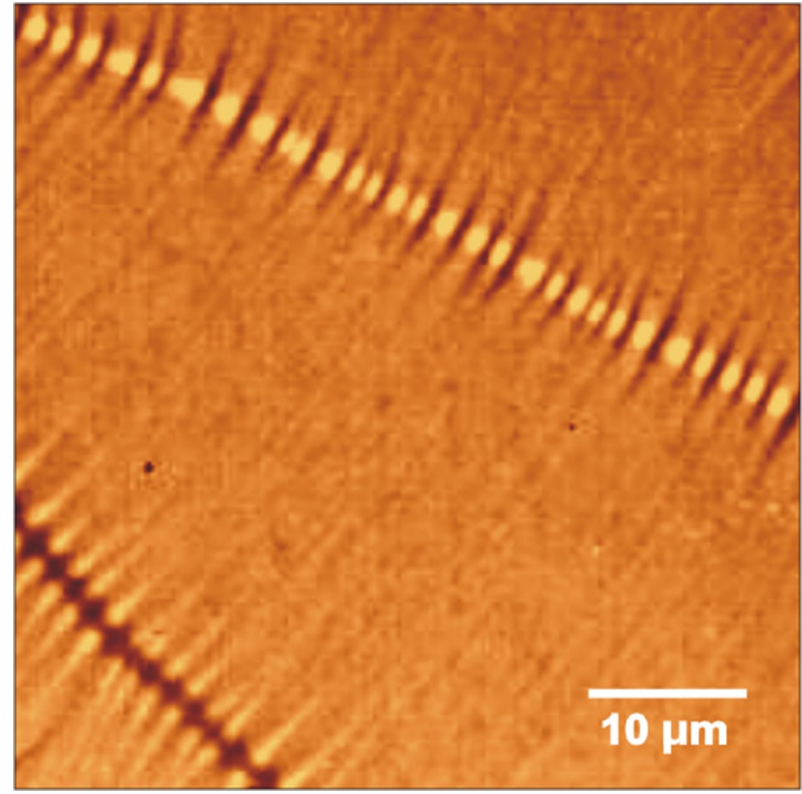

Fig. 6 Image of the magnetic microstructure of a 40 nm-thick permalloy film, taken with the Fresnel mode of TEM

easy axis $[39,42]$. The ripple structure of the magnetization is also visible in the image of a permalloy film in Fig. 6, but with smaller clarity than in the images of cobalt films 
(Fig. 5a-f). This is again due to the large difference in the magnetocrystalline anisotropy of cobalt and permalloy. The much stronger magnetic anisotropy of cobalt causes that the ripple structure of the magnetization in Fresnel images is considerably more pronounced.

The Fresnel mode of TEM senses only the inplane component of magnetization. To obtain a complementary information on the magnetization direction of the investigated cobalt films, we used also MFM to image the magnetic microstructure. The MFM method probes the stray field of the specimen and consequently is substantially sensitive to the perpendicular component of magnetization (in the case of specimens with inplane magnetization, the only sources of the stray field are the domain walls and surface defects $[43,44])$. It is worth noting here that different MFM cantilevers were used for each sample. Each cantilever used was checked before and after every measurement session to guarantee high contrast of MFM images (indicating high magnetic sensitivity of particular cantilever). The sample used in these cantilever tests was thick Co film characterized by perpendicular magnetization.

Using MFM measurements, we did not detect any domains with perpendicular magnetization component in cobalt films thinner than $50 \mathrm{~nm}$ (all recorded domains are characterized by the same MFM contrast). This changes for $50 \mathrm{~nm}$-thick films for which perpendicular magnetization shows up (see Fig. 7 which shows three representative MFM images of the magnetic domain structure recorded in $50 \mathrm{~nm}$-thick cobalt films). In regions such as that presented in Fig. 7a, the magnetic structure was observed to be composed of large irregular domains (typically from a few to $10 \mathrm{~mm}$ in size) with dominating inplane magnetization component. The large irregular domains are substantially magnetized in the plane of the film with extremely weak perpendicular magnetization component (they are displayed all nearly at the same color shade). These domains are separated by the domain walls. One of these walls seen in right part of Fig. 7a (running from its top to bottom) is characterized by the presence of four segments: dark and bright ones on each side of the domain wall (with reversed contrast with respect to the wall, see encircled areas in the image). Such a MFM image is typical for cross-tie wall [42]. In regions such as that shown in Fig. 7b, the magnetic structure was observed to consist of fine stripe domains (of the order of $100 \mathrm{~nm}$ in width) forming a maze pattern with dominating perpendicular magnetization component. This also indicates that the magnetostatic interaction between the fine domains and the tip was nonperturbing [45, 46]. Finally, the $50 \mathrm{~nm}$-thick Co films were also characterized by the presence of mixed regions characterized both by inplane and perpendicular magnetization, i.e., in Fig. 7c, the large irregular domains and fine stripe domains can be seen simultaneously.

In $60 \mathrm{~nm}$-thick films, there are also both inplane and perpendicular magnetization components, the presence of which is evidenced in the Fresnel image of Fig. $5 \mathrm{f}$ and in the MFM image of Fig. 8, respectively. However, for thicker films, i.e., 70, 80, 90, and $100 \mathrm{~nm}$-thick [47], we observed only perpendicular magnetization of the surface. Thus, the obtained results show that the transition from fully inplane to fully perpendicular magnetization takes place for film thicknesses comprised 40 and $70 \mathrm{~nm}$.

In the above context, it is worth to note micromagnetic simulations of the magnetization distribution evolution in the cobalt thickness range of few tens of nanometers, published by Kisielewski et al. [48]. It was found that the transition from inplane to perpendicular magnetization configuration takes place at the film thickness of $22 \mathrm{~nm}$. The obtained result is found to be in reasonable agreement with the experimental results reported in Refs. [12-15]. and by us.

All the studied films consist of small grains. The average grain sizes of these films are in the range from $22.0 \mathrm{~nm}$ (for $10 \mathrm{~nm}$-thick films) to $28.9 \mathrm{~nm}$ (for $60 \mathrm{~nm}$ thick films) as presented in Fig. 3. This leads to strong exchange coupling among the grains (formed usually
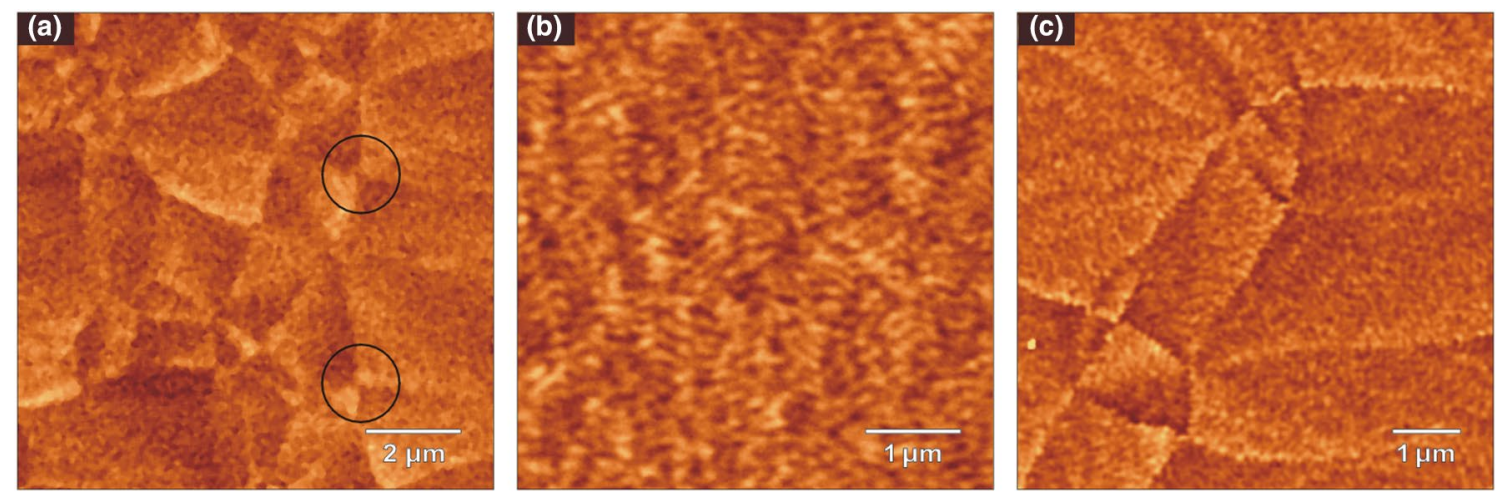

Fig. 7 MFM images of the magnetic microstructure of a $50 \mathrm{~nm}$-thick cobalt film 


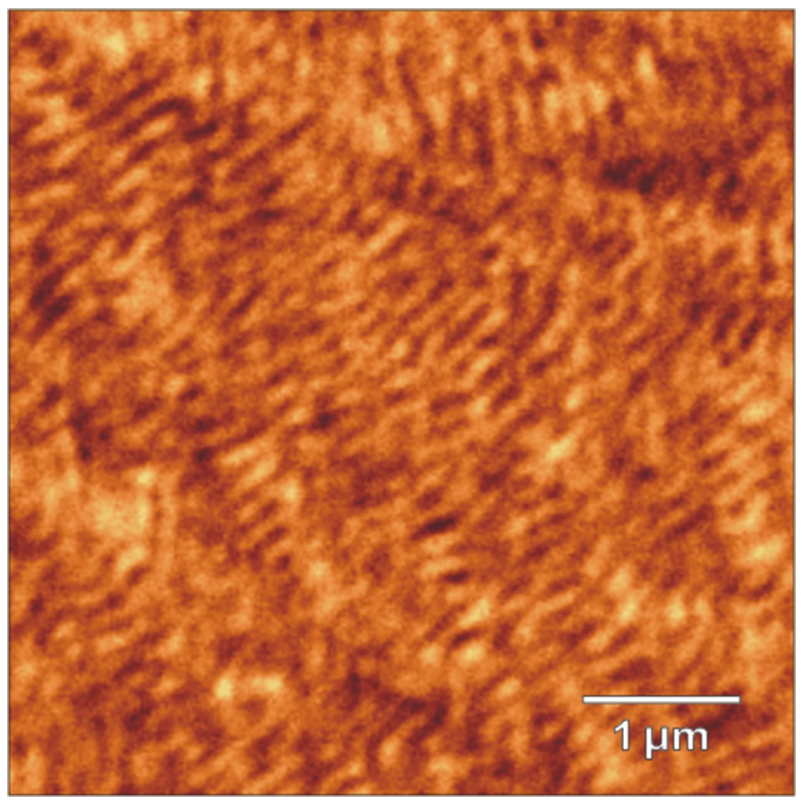

Fig. 8 MFM image of the magnetic microstructure of a 60 nm-thick cobalt film

when the average grain size is about $40 \mathrm{~nm}$ or less) [27, 49]. Thus, for the studied films, the exchange interaction is found to play a very significant role by smoothing and averaging the random distribution of the magnetocrystalline anisotropy of the individual grains.

Note that the grain size is so small that the magnetization cannot be oriented along the magnetic easy axis. It is because the exchange energy and the magnetostatic energy at the grain boundaries would become too large [50]. As a result, the total effective magnetic anisotropy is strongly reduced in comparison to the magnetocrystalline anisotropy. In consequence of the magnetic anisotropy reduction, an increase of magnetic domains width can be observed, as shown in Figs. 5a-f and 7a in which the in-plane domain structure with large domains of the order of $10 \mu \mathrm{m}$ in size can be observed in Refs [7, 8, 35, $51,52]$.

The films with thicknesses of 50 and $60 \mathrm{~nm}$ possess also magnetization component perpendicular to the film plane, represented in the form of fine stripe domains (of the order of $100 \mathrm{~nm}$ in width), as shown in Figs. $7 \mathrm{~b}$ and 8. The presence of the fine stripe domain structure means that sufficiently strong crystallographic alignment of the cobalt grains with the hexagonal axis perpendicular to the film surface is present in these films. The shape anisotropy of these films is smaller than that of the thinner films, and the perpendicular magnetocrystalline anisotropy becomes more and more significant.

\section{Conclusions}

We have performed a detailed investigation of the morphological and magnetic domain structures of nanocrystalline thin cobalt films. The films possessed thicknesses in the range from 10 to $60 \mathrm{~nm}$ and were obtained by thermal evaporation at an incidence angle of $0^{\circ}$ in a vacuum of approximately $10^{-5}$ mbar. The morphological structure of the films was observed by AFM, while their magnetic domain structure was made visible with MFM and the Fresnel mode of TEM. High spatial resolution and clear images were obtained and analyzed in detail.

The morphological structure is composed of grains. The grains have small sizes in nanometer range and are packed densely. The grains are regular in shape and generally exhibit no geometric alignment as well as no preferred elongation. The average grain size increases with increasing film thickness, from 22.0 to $28.9 \mathrm{~nm}$ as the film thickness is increased from 10 to $60 \mathrm{~nm}$. As the film thickness is increased from 10 to $40 \mathrm{~nm}$, the average surface roughness increases from 0.44 to $0.89 \mathrm{~nm}$, and for 50 and $60 \mathrm{~nm}$ thick films, it is practically the same as that for $40 \mathrm{~nm}$-thick films. The films crystallize mainly in the HCP phase of cobalt and exhibit the presence of (0002) texture.

Thanks to the application of MFM and the Fresnel mode of TEM, we could obtain a complementary information about the magnetic domain structure. In films with thicknesses in the range from 10 to $40 \mathrm{~nm}$, the domains have magnetizations in the plane of the film. Whereas in 50 and $60 \mathrm{~nm}$-thick films, there are both inplane and perpendicular magnetization components. The domains with inplane magnetization are irregular in shape and possess large sizes typically from a few to $10 \mathrm{~mm}$, and the domains with perpendicular magnetization are in the form of a fine maze stripe structure of the order of $100 \mathrm{~nm}$ in width. The large inplane domains are the result of the strongly reduced magnetic anisotropy due to a strong exchange interaction between the grains. The occurrence of the fine stripe domain pattern in 50 and $60 \mathrm{~nm}$-thick films is found to result from sufficiently strong crystallographic alignment of the cobalt grains with the hexagonal axis perpendicular to the film plane. The shape anisotropy of these films is smaller than that of the thinner films, and the perpendicular magnetocrystalline anisotropy becomes more and more significant. The transition from fully inplane to fully perpendicular magnetization takes place for film thicknesses comprised 40 and $70 \mathrm{~nm}$.

In $10 \mathrm{~nm}$-thick films, only the domain walls of Néel type occur; in 20 and $30 \mathrm{~nm}$-thick films, both Néel type and cross-tie walls are present, while in films with thicknesses in the range from 40 to $60 \mathrm{~nm}$, only cross-tie walls occur. The coexistence of Néel type and cross-tie walls in 20 and $30 \mathrm{~nm}$-thick films means that their wall energies are comparable at these film thicknesses. Considering the 
simplifications used in the theoretical works of Metlov [40, 41], the experimental data appear to confirm quite well the predictions of the theory. The cross-tie walls are characterized by small cross-tie spacing and length, due to the high magnetic anisotropy of the HCP phase of cobalt.

Acknowledgements This work was supported by the Łódź University within Research Grant B1611500000110.01.

Open Access This article is distributed under the terms of the Creative Commons Attribution 4.0 International License (http:// creativecommons.org/licenses/by/4.0/), which permits unrestricted use, distribution, and reproduction in any medium, provided you give appropriate credit to the original author(s) and the source, provide a link to the Creative Commons license, and indicate if changes were made.

\section{References}

1. U. Hartmann (ed.), Magnetic multilayers and giant magnetoresistance (Springer Science and Business Media, vol. 37, 2013)

2. D. Jiles, Introduction to magnetism and magnetic materials (CRC press Taylor \& Francis Group, 2015)

3. T. Niizeki, Y. Utsumi, R. Aoyama, H. Yanagihara, J.I. Inoue, Y. Yamasaki, H. Nakao, K. Koike, E. Kita, Appl. Phys. Lett. 103, 62407 (2013)

4. R. Streubel, J. Lee, D. Makarov, M.Y. Im, D. Karnaushenko, L. Han, R. Schäfer, P. Fischer, S.K. Kim, O.G. Schmidt, Adv. Mater. 26, 316 (2014)

5. J.J. Lee, G.Z. Xing, J.B. Yi, T. Chen, M. Ionescu, S. Li Appl. Phys. Lett. 104, 012405 (2014)

6. B.A. Assaf, T. Cardinal, P. Wei, F. Katmis, J.S. Moodera, D. Heiman, Appl. Phys. Lett. 102, 012102 (2013)

7. W. Szmaja, W. Kozłowski, K. Polański, J. Balcerski, M. Cichomski, J. Grobelny, M. Zieliński, E. Miękoś, Chem. Phys. Lett. 542, 117 (2012)

8. W. Szmaja, W. Kozłowski, K. Polański, J. Balcerski, M. Cichomski, J. Grobelny, M. Zieliński, E. Miękoś, Mater. Chem. Phys. 132, 1060 (2012)

9. W. Kozlowski, I. Piwonski, M. Zielinski, E. Miekos, K. Polanski, W. Szmaja, M. Cichomski, Appl. Phys. A 120, 155 (2015)

10. D.M. Donnet, K.M. Krishnan, Y. Yajima, J. Phys. D Appl. Phys 28, 1942 (1995)

11. M. Hehn, S. Padovani, K. Ounadjela, J.P. Bucher, Phys. Rev. B 54, 3428 (1996)

12. J. Brandenburg, R. Hühne, L. Schultz, V. Neu, Phys. Rev. B 79, 054429 (2009)

13. N. Kumar, R.W.A. Hendrikx, A.J.L. Adam, P.C.M. Planken, Opt. Express 23, 14252 (2015)

14. W.G. Wang, C.L. Chien, J. Phys. D Appl. Phys 46, 074004 (2013)

15. S. P. Dash, D. Goll, H.D. Carstanjen, Appl. Phys. A 97, 651 (2009)

16. G. Wei, H. Ge, Q. Wu, Y. Yu, X. Wang, C. Chen, Mater. Chem. Phys 118, 57 (2009)

17. S.H. Kang, K. Lee, Acta Mater 61, 952 (2013)

18. C. Moreau-Luchaire, C. Moutafis, N. Reyren, J. Sampaio, C.A.F. Vaz, N. Van Horne, K. Bouzehouane, K. Garcia, C. Deranlot, P. Warnicke, P. Wohlhüter, J.-M. George, M. Weigand, J. Raabe, V. Cros, A. Fert, Nat. Nanotech. 11, 444 (2016)
19. A. Fert, V. Cros, J. Sampaio, Nat Nanotechnol 8, 152 (2013)

20. M.D. Cooke, D.A. Allwood, D. Atkinson, G. Xiong, C.C. Faulkner, R.P. Cowburn, J. Magn. Magn. Mater. 257, 387 (2003)

21. J.N. Chapman, J. Phys. D Appl. Phys 17, 623 (1984)

22. W. Szmaja, J. Balcerski, W. Kozłowski, M. Cichomski, J. Grobelny, M. Smolny, P.J. Kowalczyk, J. Alloys Compd. 521, 174 (2012)

23. W. Szmaja, W. Kozłowski, J. Balcerski, P.J. Kowalczyk, J. Grobelny, M. Cichomski, J. Alloys Compd. 506, 526 (2010)

24. M.A. Akhter, D.J. Mapps, Y.Q. Ma Tan, A. Petford-Long, R. Doole, J. Appl. Phys 81, 4122 (1997)

25. G. Valdré, Nanostruct. Mater. 10, 419 (1998)

26. A. Hubert, R. Schäfer, Magnetic domains: the analysis of magnetic microstructures. (Springer, Berlin, 1998)

27. W. Szmaja, J. Grobelny, M. Cichomski, S. Hirosawa, Y. Shigemoto, Acta Mater. 59, 531 (2011)

28. A. Kharmouche, S.-M. Chérif, A. Bourzami, A. Layadi, G. Schmerber, J. Phys. D Appl. Phys. 37, 2583 (2004)

29. W. Gil, D. Görlitz, M. Horisberger, J. Kötzler, Phys. Rev. B 72, 134401 (2005)

30. M.F. Chioncel, H.S. Nagaraja, F. Rossignol, P.W. Haycock, J. Magn. Magn. Mater. 313, 135 (2007)

31. A.A. Karimpoor, U. Erb, K.T. Aust, G. Palumbo, Scripta Mater. 49, 651 (2003)

32. K. Itoh, K. Okamoto, T. Hashimoto, J. Magn. Magn. Mater. 190, 176 (1998).

33. T.G. Woodcock, K. Khlopkov, A. Walther, N.M. Dempsey, D. Givord, L. Schultz, O. Gutfleisch Scripta Mater. 60, 826 (2009)

34. M. Gallaugher, N. Brodusch, R. Gauvin, R.R. Chromik, Ultramicroscopy 142, 40 (2014)

35. W. Szmaja, in Advances in imaging and electron physics, Ed. by P.W. Hawkes (Elsevier, Amsterdam, Vol. 141, 2006), p. 175

36. H.-N. Lin, Y.H. Chiou, B.-M. Chen, H.-P.D. Shieh, C.-R. Chang, J. Appl. Phys. 83, 4997 (1998)

37. E.E. Huber Jr., J.B.D.O. Smith, Goodenough. J. Appl. Phys. 29, 294 (1958)

38. S. Middelhoek, J. Appl. Phys. 34, 1054 (1963)

39. A. Gentils, J.N. Chapman, G. Xiong, R.P. Cowburn, J. Appl. Phys. 98, 053905 (2005)

40. K.L. Metlov, Appl. Phys. Lett. 79, 2609 (2001)

41. K.L. Metlov, J. Low Temp. Phys. 139, 207 (2005)

42. M. Löhndorf, A. Wadas, H.A.M. van den Berg, R. Wiesendanger, Appl. Phys. Lett. 68, 3635 (1996)

43. P. Grütter, H.J. Mamin, D. Rugar, Scanning tunneling microscopy II, Ed. by R. Wiesendanger, H.-J. Güntherodt (Springer, Berlin 1992), p. 151

44. U. Hartmann, Annu. Rev. Mater. Sci. 29, 53 (1999)

45. W. Szmaja, J. Grobelny, M. Cichomski, Appl. Phys. Lett. 85, $2878(2004)$

46. M. Cichomski, W. Szmaja, Appl. Phys. A 102, 339 (2011)

47. W. Kozłowski, J. Balcerski, W. Szmaja, J. Magn. Magn. Mater. 423, 256-261 (2017)

48. M. Kisielewski, A. Maziewski, V. Zablotskii, J. Magn. Magn. Mater. 316, 277 (2007)

49. G.C. Hadjipanayis, J. Magn. Magn. Mater. 200, 373 (1999)

50. R. Schäfer, Nanoscale magnetic materials and applications, Ed. by J.P. Liu, E. Fullerton, O. Gutfleisch, D.J. Sellmyer (Springer, Dordrecht 2009), p. 275

51. Y. Gao, J. Zhu, Y. Weng, B. Han, Appl. Phys. Lett. 74, 1749 (1999)

52. Y.K. Fang, X. Yin, R. Skomski, H. Cheng, K. Song, M. Zhu, Z. Guo, W. Li, S.H. Liou, Scripta Mater. 111, 72 (2016) 